\title{
Caracterización del modelo de implantación urbana y ocupación del territorio de la antigua Zona del Canal. Caso de estudio Fuerte Clayton
}

\author{
Arosemena, Graciela \\ Universidad de Panamá/SNI \\ Panamá, Panamá \\ graciela.arosemena@up.ac.pa \\ Alba, Almyr \\ Universidad de Panamá \\ Panamá, Panamá \\ almyr.alba@up.ac.pa \\ Stapf, María \\ Universidad de Panamá \\ Panamá, Panamá \\ maria.stapf@up.ac.pa \\ Carceller, Xavier \\ Independiente \\ xcr@coac.cat

\section{Abstract}

This study aims to understand the influence of North American urbanism in the Canal Zone, to determine what urbanization processes occurred and the implications this had on human health, natural resources and biodiversity, through the case of Fort Clayton. To do this, the influence that the North American hygienist urbanism of the 19th century had on the urban implantation model of the Canal Zone is identified, and the common elements are identified. And how the strategies to control mosquito-borne diseases were reflected in urban planning and in the impact on the territory.

The study is based on the environmental assessment of Fort Clayton, determined by the two fundamental aspects that determine the environmental impact of an urban settlement. The first the suitability of the location of the site, and the second the urban structure of the city model that is adopted.

Concluding that the location of Fort Clayton from the environmental and sanitary point 
of view was not suitable, and the continuous tasks of territorial control of the mosquito breeding sites over time show it. So the ecological matrix of the territory was altered in order to remedy an inappropriate location. On the other hand, the adaptation of the urban layout to the topography and the systematized use of drains in the towns and forts of the Canal Zone, was a usual practice, with obvious references to the health postulates of the 19th century urban planning.

Keywords: Urban history, environment, mosquitoes' control, Canal Zone, Fort Clayton

\section{Resumen}

Este estudio tiene como objetivo comprender la influencia del urbanismo norteamericano en la Zona del Canal, determinar qué procesos de urbanización se dieron y las implicaciones ello tuvo en la salud humana, los recursos naturales y la biodiversidad, a través del caso del Fuerte Clayton. Para ello se identifica la influencia que tuvo el urbanismo higienista norteamericano del siglo XIX sobre el modelo de implantación urbana de la Zona del Canal, e identificar los elementos en común. Y cómo las estrategias de control de las enfermedades transmitidas por los mosquitos se reflejaron en el urbanismo y en el impacto sobre el territorio.

El estudio se basa en la valoración ambiental del Fuerte Clayton, determinado por los dos aspectos fundamentales que definen el impacto ambiental de un asentamiento urbano. El primero la idoneidad de la localización del sitio, y el segundo la estructura urbanística del modelo ciudad que se adopta.

Concluyendo que la localización del Fuerte Clayton desde el punto de vista ambiental y sanitario no fue idóneo, y las tareas continuas de control territorial de los criaderos de mosquito a lo largo del tiempo lo evidencian. De forma que la matriz ecológica del territorio fue alterada con el fin de remediar una ubicación improcedente. Por otro lado la adaptación del trazado urbano a la topografía y el uso sistematizado de drenajes en los poblados y fuertes de la Zona del Canal, fue una práctica usual, con evidentes referencias a los postulados sanitarios del urbanismo del siglo XIX.

Palabras claves: Historia urbana, medio ambiente, control de mosquitos, Zona del Canal, Fuerte Clayton. 


\section{INTRODUCCIÓN. ANTECEDENTES DEL URBANISMO HIGIENISTA NORTEAMERICANO}

Uno de los aspectos sanitaros que probablmente más influyó en el modelo urbano que surgió en el siglo XIX en norteamerica, fue la búsqueda de una solución al hacinamiento, y la prevención de formación de charcos de agua lluvia en las calles, los cuales se consideraba un grave problema sanitario [1][2].

Con el fin de comprender la incidencia de malaria y fiebre amarilla en la ciudad de Nueva York, el ingeniero sanitario Edgard Viele elabora un mapa de Nueva York en 1874 representando la topografía y los cuerpos de agua naturales y los que fueron bloqueados por la edificación de la ciudad. Viele identificó de forma empírica cruzando las estadísticas de enfermedades con el mapa, que las zonas más deprimidas, que acumulan más agua lluvia, y las zonas próximas a los ríos bloqueados, había una mayor incidencia de enfermedades y muertes [2].

Como Viele numerosos profesionales de la recién nacida disciplina sanitaria, se habían lanzado en la búsqueda de la etiología de las enfermedades, que, aunque apoyados en la teoría miasmática, determinaron que las mejoras de las condiciones ambientales de las ciudades reducían la incidencia de enfermedades [3].

A pesar de que no se comprendía el origen real de las enfermedades contagiosas, el conocimiento empírico inició una serie de cambios urbanos que sentarían las bases del urbanismo moderno [4]. Y uno de los aspectos que se intentaba resolver fue la prevención de la acumulación de agua, que estaba entonces asociada a la incidencia de malaria.

Ya en 1867 el ingeniero sanitario Waring había determinado la necesidad de estudiar la topografía y los cuerpos de agua de un terreno antes de intervenirlo. Y que de ser necesario debían construirse drenajes en el terreno y drenajes para secar los cuerpos de agua.

Este ing sanitario fue amigo del arquitecto paisajista que mayor influencia tendría en el urbanismo norteamericano del S XIX, Frederick Olmsted; quien asumió todos los conceptos sanitarios y los aplicó al planeamiento Olmsted dedicó grandes esfuerzos para resolver esos problemas. Determinando que se debía evitar urbanizar en terrenos bajos e inundables [5]. Y del estudio de la topografía y la prevención de la acumulación de agua lluvia Olmsted desarrolla un trazado sinuoso de calles, adaptadas a las curvas de nivel, para facilitar el drenaje [6]. Este es el origen del trazado ampliamente utilizado en la Zona del Canal.

Por otro lado, sus postulados, manifestaban una fuerte oposició a lo que él llamaba "pasada de moda" forma compacta de edificar las ciudades la cual estaba asociada a un mayor riesgo de contagios. Y en cambio abogaba por descomprimir la ciudad, y adoptar una distribución de la edificación dejando amplios espacios entre ellas. Ello en terminos habitacionales significó la entrada de la vivienda aislada en la planificación de los suburbios a partir de mediados del siglo XIX. De forma que, para completar su modelo urbano sano, 
Olmsted, abogó por una baja densidad, viviendas unifamiliares aisladas, amplios espacios abiertos.

De forma que en lo que quedaba del siglo XIX y principios del XX, la manera de concebir las ciudades en los Estados Unidos estuvo marcada por la baja densidad de población, y en especial los suburbios, continuaron desarrollandose guardando distancias entre las viviendas, y con grandes espacios abiertos, y parques.

De forma similar ocurrió en la Zona del Canal. Y en el caso concreto del conjunto residencial del Fuerte Clayton que se estudia, fueron desarrolladas edificios bifamiliares separadas a una distancia de XX, y un amplio espacio verde central, que fue pensado como un playground.

La caracterización de la implantación urbana al territorio de la antigua Zona del Canal ni la influencia del urbanismo norteamericano no han sido identificados en detalle. El presente artículo determina cómo fue el proceso de urbanización en la Zona y qué implicaciones tuvo en la salud humana, los recursos naturales y la biodiversidad, a través del caso del Fuerte Clayton.

\section{MÉTODO}

En general, el estudio busca comprender qué llevó a los norteamericanos a desarrollar el modelo urbano aplicado, y las implicaciones ambientales que ello pudo tener. Además establecer la influencia que tuvieron las medidas de control del mosquito en el Fuerte sobre el territorio.

Determinando cómo la experiencia del urbanismo higienista del siglo XIX se va a reproducir en la Zona del Canal se consideran en los siguientes ámbitos:

- El manejo del territorio

- La implantación urbana al terreno

Determinando además cómo estas se vería reforzadas por las estrategias de la lucha contra el mosquito transmisor de la malaria, el principal enemigo del Canal y de los planes de poblamiento de la Zona.

El estudio se basa en la valoración del Fuerte Clayton, determinado por los dos aspectos fundamentales que determinan el impacto ambiental de un asentamiento urbano. El primero la idoneidad de la localización del sitio, y el segundo la estructura urbanística del modelo ciudad que se adopta. Entendiendo el impacto ambiental como las consecuencias que tienen sobre la biodiversidad, el paisaje y la seguridad de las personas.

\section{A. Preexistencias ambientales}

Para valorar la idoneidad de la localización del Fuerte Clayton, cabe determinar brevemente las preexistencias ambientales de la región pacífica en donde se ubicó el 
Fuerte y el trazado del Canal. Dicho territorio estaba formada por tierras bajas cenagosas asociadas al valle del río Grande [7] columna vertebral del Canal. Y el área en donde se ubicaría Clayton se encontraba en la confluencia del río Grande con el río Cárdenas, también un terreno bajo susceptible a inundaciones, en donde se encontraba el pequeño poblado de Río Grande.

Con los franceses se inician las obras del Canal y además un proceso de transformación del territorio del Río Grande. Tal como se aprecia en la sección de los avances del Canal para 1891, ya había comenzado a dragarse parte del lineamiento entre Corozal y Miraflores [8] Pero en realidad el terreno futuro del Clayton no parece haber sido intervenido por los franceses. Las consecuencias de la alteración del terreno ligadas a las obras del canal, propició la formación charcos que se convirtieron en criaderos de mosquitos anopheles [7]. Y con el posterior abandono del proyecto francés, y de toda su maquinaria se incrementaron los criaderos. Cuando llegaron los norteamericanos en 1904 el problema de los mosquitos ya estaba instalado en el territorio, y empeoraría aún más con la continuación de las obras del Canal [9].

\section{B. Valoración de la idoneidad de la localización del Fuerte Clayton}

Antes de valorar la idoneidad de la localización del Fuerte, analizamos la problemática territorial de los mosquitos de la Zona. Poco antes de iniciar el Canal norteamericano, había sido identificado el mosquito Anopheles como vector de la malaria. Y los entomólogos del Canal se lanzaron a una labor titánica, determinado que las condiciones territoriales existentes a lo largo de las obras del Canal, qye propiciaban los criaderos de mosquito [7] [10]:

1. Tierras bajas

2. El clima lluvioso

A pesar de las estrategias urbana contra la malaria desarrolladas por sanitarios del Canal de forma temprana en los primeros poblados de la Zona, para 1911 la malaria afectaba los poblados de Corozal y Ancón, debido a unas ciénagas naturales y artificiales en donde se habían encontrado criaderos de mosquitos. Los mismos fueron drenados con numerosas canalizaciones, una estrategia con claros referentes al siglo XIX, y en otros casos eran rellenados [7]. Pero llamó la atención la distancia que debían recorrer los mosquitos desde los criaderos hasta llegar a los poblados.

Por ello fueron realizados expermientos para comprender los hábitos de vuelo de los mosquitos, identificando que la mayoría volaba 1500 metros de distancia [7]. Al final, el punto importante a decidir si intervenir en una ciénaga o no, era la longitud de vuelo de las diferentes especies en la medida en que afectaban a la transmisión del paludismo.

Entonces, el terreno bajo seleccionado para ubicar el Fuerte era en gran parte inundable, 
y estaba rodeado de otras tierras bajas que fácilmente podían albergar criaderos de mosquitos.

Con lo cual la etapa preliminar a la ocupación urbana del Fuerte Clayton, fue el relleno de las tierras inundables con material de las excavaciones del Canal.

A partir de 1919 se inician labores previas a la construcción del Fuerte [11] similares a las aplicadas en el siglo XIX, para reducir el estancamiento de aguas, y prevenir la malaria. Fueron ejecutados trabajos en el río Cárdenas, colindante al Fuerte, que se había convertido en criadero de mosquitos, y se drenaron otros cuerpos de agua. Y a lo largo de las etapas constructivas del Fuerte, las calles se trazaron adaptándose a las curvas de nivel, como en el urbanismo sanitario del siglo XIX.

El diseño y la obra inicial del Fuerte, se realizó integrando el alcantarillado, los drenajes de agua lluvia, así como la nivelación del terreno, con el fin de prevenir criaderos de mosquitos [11].

La lucha contra la malaria una vez finalizado el Canal continuó, y en el propio Fuerte, los casos de malaria se dieron incluso hasta finales de la década de los años 1930s.

Los hábitos del mosquito responsable del $70 \%$ de los casos de malaria del sector Pacífico, el anopheles albimanus [7], influyó en la forma en que se intervino en el territorio, ya que el mismo no vive en zonas urbanas, sino que viaja varios kilómetros hasta ellas para alimentarse.

Es así cómo del estudio continuo del mosquito y la vigilancia de los criaderos de mosquito en el territorio permitió entender porqué se daban los brotes de malaria en el Fuerte Clayton, para intervenir. De forma que podemos indicar que el control territorial del mosquito provocó que el impacto del Fuerte Clayton en realidad se extendía en un radio de un kilómetro y medio, según el mapa de saneamiento del sector Pacífico [12], lo que puede denominarse una huella urbana sanitaria.

Las dificultades en el control de la malaria en el Fuerte se tradujeron en el drenaje de ciénagas, y dado que los mosquitos podían criar en aguas estancadas de riachuelos y ríos por el crecimiento vegetal de sus márgenes, se realizaban podas continuas de los bordes, $Y$ ya que los sanitarios concebían que todo cuerpo de agua era un drenaje las quebradas eran convertidas en canalizaciones de cemento [7].

\section{Estructura urbana aplicada}

El modelo urbano que fue aplicado a lo largo de todas sus etapas, en esencia no es muy diferente que el desarrollado por Frederick Olmsted en el primer suburbio planificado de Estados Unidos. Utilizando una muy baja densidad, tipología de edificación aislada, distancia entre ellas, y grandes espacios abiertos.

A nivel territorial, se desarrolló un sistema poco compacto, disperso y disgregado; lo cual 
compromete por un lado un alto consumo de suelo para ser urbanizado, y por otro es un modelo que depende del vehículo motorizado para la movilidad obligada, incrementando el consumo de combustible.

\section{RESULTADOS}

Consecuencias ambientales del modelo territorial y estructura urbana del Fuerte Clayton

Las estrategias de prevención de criaderos de mosquito a nivel territorial consistente en la poda de los márgenes de los cuerpos de agua provocaron la pérdida de ecosistemas como humedales y bosques de ribera.

Por otro lado, las canalizaciones de concreto de las quebradas se extendieron a todo Clayton, como se puede apreciar en el extracto del mapa de drenajes de 1969. Ello debió afectar los flujos naturales del agua afectando los movimientos de la fauna acuática.

En relación a la conectividad ecológica o capacidad del territorio para permitir el desplazamiento de especies de fauna, es impactada por la estructura urbana de Clayton. El carácter disgregado de la mancha urbana de Clayton, fragmenta el territorio y dificulta la movilidad de la fuana terrestre.

Pero además, la propia dispersión genera grandes espacios abiertos verdes, y manchas de bosque dentro del tejido urbano, lo que sumado a la diversidad de especies de árboles urbanos, hacen de Clayton un entorno propicio para atraer la fauna. En donde las aves son las más beneficiadas, sin embargo, la movilidad de la fauna de otros vertebrado como los mamíferos es limitada.

\section{CONCLUSIONES}

El peso de la estrategia militar de defensa del Canal pesaba más que cualquier otro factor en las decisiones de ubicación de las reservas militares, y la defensa de las esclusas de Miraflores forzaron una localización territorial del Fuerte Clayton no idónea desde el punto de vista ambiental y sanitario. Las tareas continuas a lo largo del tiempo del control territorial de los criaderos de mosquito lo evidencian. Es debido a ello que el impacto del poblamiento del sector Pacífico de la Zona del Canal sobre el territorio no estaba suscrito únicamente a la huella urbana, sino que las medidas de control de criaderos de mosquito a nivel territorial definieron una huella urbana sanitaria que iba mucho más allá de los limites de los poblados, lo que supuso la pérdida de ecosistemas y la transformación de la red hídrica natural.

Paralelamente vemos cómo la adaptación del trazado urbano a la topografía y el uso sistematizado de drenajes en los poblados y fuertes de la Zona del Canal, tuvo como objetivo evitar la formación de charcos y criaderos de mosquito, con evidentes referencias a los postulados sanitarios del urbanismo del siglo XIX. 
Y aunque los urbanistas del siglo XIX nunca comprendieron la verdadera relación entra la acumulación de agua y las enfermedades y muertes asociadas a la malaria y fiebre amarilla, la observación les permitió establecer una correlación. A pesar de ello sus soluciones dentro del tejido urbano para prevenir charcos no estaban mal encaminadas, y las mismas no solo fueron aplicadas como medida de control de los criaderos de mosquito en la Zona del Canal, sino que incluso en la actualidad son vigentes.

Desde el punto de vista de la estructura urbana, el modelo que fue implantado, aunque estaba orientado a la prevención de enfermedades, significó un alto consumo de suelo, y paradójicamente, aunque no fue diseñado con esa intención, los grandes espacios abiertos y la presencia de una arborización urbana muy diversa, propició la atracción de la fauna.

\section{Referencias}

[1] Waring, George. "Draining for Profit, and Draining for Health". New York: Entered according to Act of Congress, in the year 1867, by Orange Judd \& Co.

[2] Viele, Egbert. "Council of Hygiene and Public Health of the Citizens Association". New York: Ferd. Mayer \& Co. Litographers, 1865

[3] Taylor, Dorceta. "The Environment and the People in American cities, 1600s-1900s". Duke University Press Books, 2009

[4] Szczygiel, Bonj, y Robert Hewitt. "Nineteenth-Century Medical Landscapes: John Rauch, Frederick Olmsted and the Search of Salubrity". Bulletin of the History of Medicine (The John Hopkins University Press) Winter 2000, vol. 74, No. 4: 708-734.

[5] Olmsted, Frederick Law, y et al. 1871. "Report to the Staten Island Improvement Commission of a Preliminary Scheme of Improvements".

[6] Peterson, Jon. 1979. "The Impact of Sanitary Reform upon American Urban Planning, 1840-1890". Journal of Social HIstory (Oxfor University Press) 13 (1): 83-103.

[7] Le Prince, Joseph, A.J Orenstein, y L.O Howard. 1916. "Mosquito Control in Panama. The eradication of Malaria and Yellow Fever in Cuba and Panama". G.P Putnam's sons. New York and London. The Knickerbocker press.

[8] Mission de Lucien N.B Wyse. "Canal Interocéanique de Panama". Gallica, Biblioteque Nationale de France, 1891

[9] Sutter, P. "El control de los zancudos en Panamá: los entomólogos y el cambio ambiental durante la construcción del Canal". Historia Crítica 67-90, 2005

[10] Gorgas, William Chief Sanitary Officer. "US Sanitation in Panama. Appleton and Company", 1915

[11] "Annual Report of the Governor of the Panama Canal for the Fiscal year Ended June 30", 1920. Washington: Governement printing office.

[12] Fisher. Report of Health Department of the Panama Canal for the Calendar Year 1928. Mont Hope, Canal Zone: The Panama Canal Press, 1928 


\section{Autorización y Licencia CC}

Los autores autorizan a APANAC XVIII a publicar el artículo en las actas de la conferencia en Acceso Abierto (Open Access) en diversos formatos digitales (PDF, HTML, EPUB) e integrarlos en diversas plataformas online como repositorios y bases de datos bajo la licencia CC:

Attribution-NonCommercial-ShareAlike 4.0 International (CC BY-NC-SA 4.0) https://creativecommons. org/licenses/by-nc-sa/4.0/.

Ni APANAC XVIII ni los editores son responsables ni del contenido ni de las implicaciones de lo expresado en el artículo. 